\title{
Laser Light-Scattering Characterization of a Polymer Mixture Made of Individual Linear Chains and Clusters
}

\author{
Chi Wu,* Mohammad Siddiq, and Ka Fai Woo
}

Department of Chemistry, The Chinese University of Hong Kong, Shatin, N.T., Hong Kong

Received February 22, 1995; Revised Manuscript Received April 28, $1995^{\circ}$

\begin{abstract}
The characterization of a polymer mixture made of individual linear chains and clusters is often hindered by the presence of a small amount of high molecular weight clusters. Here, an analytical method combining both static and dynamic laser light scattering (LLS) was utilized to characterize such polymer mixtures. The LLS method was carefully verified by using a set of polymer mixtures made of two polystyrenes, in which the two polystyrenes have a very large difference in their molecular weights and the higher molecular weight polystyrene was used as an imitation of the clusters. We have successfully applied this LLS method in the study of PES-C (phenolphthalein poly(ether sulfone)), a newly developed high-performance thermoplastic resin. The PES-C sample contains about $6 \%$ high molecular weight clusters by weight. The large clusters can be removed by a filter with a smaller pore size. The average molecular weight of the clusters is $\sim 15$ times higher than that of the linear PES-C chains. The PES-C linear chain has a random coil conformation in chloroform at room temperature, while the structure of the clusters seems to be more compact. This LLS method should also be very useful in the study of aggregation or association in a polymer or colloid system as long as the large "clusters" are reasonably stable in time.
\end{abstract}

\section{Introduction}

A small amount of high molecular weight polymer clusters might sometimes be produced in a given polymerization due to chain transfer or branching, e.g. cross-linking in the epoxy curing process. ${ }^{1}$ When this happens, the final product will be a polymer mixture of individual linear chains and clusters. This small amount of high molecular weight clusters can greatly affect the rheological properties and its ultimate applications. However, the high molecular weight clusters are often overlooked in some conventional analytical methods, ${ }^{2}$ such as in size exclusion chromatography, because the amount of the clusters is too small to be detected in terms of weight or number. On the other hand, this small amount of the clusters has a larger scattering power and leads to a false apparent weight-average molecular weight $M_{\mathrm{w}, \text { app }}$ in static laser light scattering (LLS).

According to the LLS theory, ${ }^{3,4}$ the weight-average molecular weight $M_{\mathrm{w}}$ can be related to the excess absolute time-averaged scattered intensity over solvent (known as the Rayleigh ratio $\left[R_{\mathrm{vv}}(q)\right]$ ) by

$$
\frac{K C}{R_{\mathrm{vv}}(q)} \cong \frac{1}{M_{\mathrm{w}}}\left(1+\frac{1}{3}\left\langle R_{\mathrm{g}}{ }^{2}\right\rangle q^{2}\right)+2 A_{2} C
$$

where $K=4 \pi^{2} n^{2}(\partial n / \partial C)^{2} /\left(N_{\mathrm{A}} \lambda_{0}{ }^{4}\right)$ with $N_{\mathrm{A}}, n$, and $\lambda_{0}$ being Avogadro's number, the solvent refractive index, and the wavelength of light in vacuo, respectively; $C$ is the total polymer concentration $(\mathrm{g} / \mathrm{mL}) ; q=\left(4 \pi n / \lambda_{0}\right) \sin (\theta / 2)$ with $\theta$ being the scattering angle; $\left\langle R_{\mathrm{g}}{ }^{2}\right\rangle^{1 / 2}$ (written as $\left\langle R_{\mathrm{g}}\right\rangle$ ) is the $z$-average radius of gyration; and $A_{2}$ is the second virial coefficient. If there exist some clusters with a high weight-average molecular weights, $M_{\mathrm{w}}$ will be an apparent weight-average molecular weight $M_{\mathrm{w}, \text { app }}$ and

${ }^{\otimes}$ Abstract published in Advance ACS Abstracts, June 1, 1995.

$$
\begin{aligned}
& M_{\mathrm{w}, \mathrm{app}}= \frac{\int_{0}^{\infty} M f_{\mathrm{w}}(M) \mathrm{d} M}{\int_{0}^{\infty} f_{\mathrm{w}}(M) \mathrm{d} M}=\frac{\int_{0}^{\infty} M C(M) \mathrm{d} M}{\int_{0}^{\infty} C(M) \mathrm{d} M} \\
&= \frac{\int_{0}^{\alpha} M C(M) \mathrm{d} M}{\int_{0}^{\alpha} C(M) \mathrm{d} M} \frac{\int_{0}^{\alpha} C(M) \mathrm{d} M}{\int_{0}^{\infty} C(M) \mathrm{d} M}+ \\
& \frac{\int_{\alpha}^{\infty} M C(M) \mathrm{d} M}{\int_{\alpha}^{\infty} C(M) \mathrm{d} M} \frac{\int_{\alpha}^{\infty} C(M) \mathrm{d} M}{\int_{0}^{\infty} C(M) \mathrm{d} M} \\
&=M_{\mathrm{w}, \mathrm{L}} x_{\mathrm{L}}+M_{\mathrm{w}, \mathrm{H}} x_{\mathrm{H}}
\end{aligned}
$$

where the subscripts " $\mathrm{L}$ " and " $\mathrm{H}$ " denote low molecular weight linear polymer chains and high molecular weight clusters, respectively; $\alpha$ is the cutoff molecular weight between the linear chains and clusters; $f_{\mathrm{w}}(M)[\propto C(M)]$ represents the weight distribution; $\int_{0}^{\alpha} C(M) \mathrm{d} M$ and $\int_{\alpha}^{\infty} C(M) \mathrm{d} M$ are the concentrations $\left(C_{\mathrm{L}}\right.$ and $\left.C_{\mathrm{H}}\right)$ of the linear chains and clusters, respectively; $x_{\mathrm{L}}\left(=C_{\mathrm{I}} / C\right)$ and $x_{\mathrm{H}}\left(=C_{\mathrm{H}} / C\right)$ are the weight fractions; and $x_{\mathrm{L}}+x_{\mathrm{H}}=1$. Equation 2 clearly shows that if $M_{\mathrm{w}, \mathrm{H}} \gg M_{\mathrm{w}, \mathrm{L}}$, the $M_{\mathrm{w}}$ measured from static LLS will be seriously distorted by the presence of a very small amount of high molecular weight clusters. Therefore, it is very important to have a reliable analytical method for the characterization of lower molecular weight linear chains in the presence of high molecular weight clusters.

It is the study of phenolphthalein poly(ether sulfone) (PES-C) that brought us into this kind of "chain-andcluster" mixture. PES-C as a new kind of highperformance thermoplastic resin has recently been developed to substitute for poly(ether ether ketone) (PEEK) because the process of PES-C is much easier in comparison with that of PEEK. The previous static LLS studies of a similar system showed that the $M_{\mathrm{w}}$ of PES-C is higher than the expected value of 100000 for polymers made from polycondensation, ${ }^{5}$ which implies the existence of some unexpected high molecular weight 
species in PES-C. Moreover, the high glass transition temperature of PES-C also leads us to believe the existence of some high molecular weight clusters in PES-C. In order to prove it, we adopted a method of combining both static and dynamic LLS to characterize PES-C. This LLS method has recently been used to characterize a block ionomer in the presence of small amounts of ionomer aggregates. ${ }^{6}$ Before using this LLS method, first we would like to prove the feasibility of applying this method to the characterization of a polymer mixture made of individual linear chains and clusters.

\section{Basic Principles}

In dynamic LLS, a precise intensity-intensity time correlation function $G^{(2)}(t, q)$ in the self-beating mode is measured, which has the following form ${ }^{7,8}$

$$
G^{(2)}(t, q)=\langle I(t, q) I(0, q)\rangle=A\left[1+\beta\left|g^{(1)}(t, q)\right|^{2}\right]
$$

where $A$ is a measured baseline, $\beta$ is a parameter depending on the coherence of the detection, $t$ is the delay time, and $g^{(1)}(t, q)$ is the normalized first-order electric field time correlation function. For a polydisperse sample, $g^{(1)}(t, q)$ is related to the line-width distribution $G(\Gamma)$ by

$$
g^{(1)}(t, q)=\left\langle E(t, q) E^{*}(0, q)\right\rangle=\int_{0}^{\infty} G(\Gamma) \mathrm{e}^{-\Gamma t} \mathrm{~d} \Gamma
$$

where the line width $\Gamma$ usually depends on both $C$ and $\theta$. This dependence can be expressed $\mathrm{as}^{9}$

$$
\Gamma / q^{2}=D=D_{0}^{0}\left(1+k_{\mathrm{d}} C\right)\left(1+f\left\langle R_{\mathrm{g}}{ }^{2}\right\rangle q^{2}\right)
$$

where $D$ is the diffusion coefficient, the superscript " 0 " and subscript " 0 " denote $C \rightarrow 0$ and $q \rightarrow 0$, respectively, $f$ is a dimensionless constant, and $k_{\mathrm{d}}$ is the diffusion second virial coefficient. $G(\Gamma)$ in eq 4 can be obtained from the Laplace inversion of the measured time correlation function. Further, $G(\Gamma)$ can be converted into $G(D)$. On the basis of eqs 1 and 4 , at $t \rightarrow 0$ and $C \rightarrow 0$, we have

$$
g^{(1)}(0, q)=\int_{0}^{\infty} G(\Gamma) \mathrm{d} \Gamma \propto R_{\mathrm{vv}}(q) \propto M_{\mathrm{w}} C
$$

It shows that the integrated area $(A)$ under $G(\Gamma)$ (or $G(D))$ is proportional to the excess scattered intensity over solvent. For a polymer mixture of linear chains and clusters, if there exist two distinct peaks in $G(D)$, denoted as $G_{\mathrm{L}}(D)$ and $G_{\mathrm{H}}(D)$, the area ratio $A_{\mathrm{r}}$ of these two peaks can be written as

$$
A_{\mathrm{r}}=\frac{A_{\mathrm{L}}}{A_{\mathrm{H}}}=\frac{\int_{0}^{\gamma} G_{\mathrm{L}}(D) \mathrm{d} D}{\int_{\gamma}^{\infty} G_{\mathrm{H}}(D) \mathrm{d} D}=\frac{M_{\mathrm{w}, \mathrm{L}} C_{\mathrm{L}}}{M_{\mathrm{w}, \mathrm{H}} C_{\mathrm{H}}}=\frac{M_{\mathrm{w}, \mathrm{L}} x_{\mathrm{L}}}{M_{\mathrm{w}, \mathrm{H}} x_{\mathrm{H}}}
$$

where as before the subscripts " $\mathrm{L}$ " and " $\mathrm{H}$ " denote low molecular weight linear chains and high molecular weight clusters, respectively, and $\gamma$ is the cutoff translational diffusion coefficient between $G_{\mathrm{L}}(D)$ and $G_{\mathrm{H}}(D)$. On the basis of eqs 2 and 7, a combination of $M_{\mathrm{w} \text {,app }}$ from static LLS and $A_{\mathrm{r}}$ from dynamic LLS will lead to $M_{\mathrm{w}, \mathrm{L}} x_{\mathrm{L}}$ and $M_{\mathrm{w}, \mathrm{H}} x_{\mathrm{H}}$. In principle, by knowing any one of the four parameters ( $M_{\mathrm{w}, \mathrm{L}}, M_{\mathrm{w}, \mathrm{H}}, x_{\mathrm{L}}$, and $x_{\mathrm{H}}$ ), we will be able to determine the remaining three parameters.

\section{Experimental Section}

Samples. Three narrowly distributed polystyrene standards $\left(M_{\mathrm{w}} / M_{\mathrm{n}}<1.05\right)$ from Polymer Laboratories Ltd., were used without further purification. Static LLS results confirmed that the weight-average molecular weights of these polystyrene standards are $1.20 \times 10^{5}, 3.00 \times 10^{5}$, and $5.94 \times$ $10^{6}$, respectively. Three polymer mixtures were made from two of these three polystyrene standards with different macroscopic weight ratios, so that the ratios of the scattered light intensity between the two polystyrenes in the mixture were $\sim 1: 1, \sim 1: 2$, and $\sim 2: 1$. These mixtures are denoted as $\mathrm{MX}-11, \mathrm{MX}-12$, and MX-21 hereafter. In these mixtures, polystyrene with a higher molecular weight acted as the "cluster". The data for these mixtures, such as $M_{\mathrm{w}, \mathrm{L}}^{\mathrm{m}}, M_{\mathrm{w}, \mathrm{H}}^{\mathrm{m}}$, $x_{\mathrm{L}}^{\mathrm{m}}, A_{\mathrm{r}}^{\mathrm{c}}$, and $M_{\mathrm{w}}^{\mathrm{c}}$, are listed in Table 1 .

Phenolphthalein poly(ether sulfone) (PES-C) with the following chemical structure

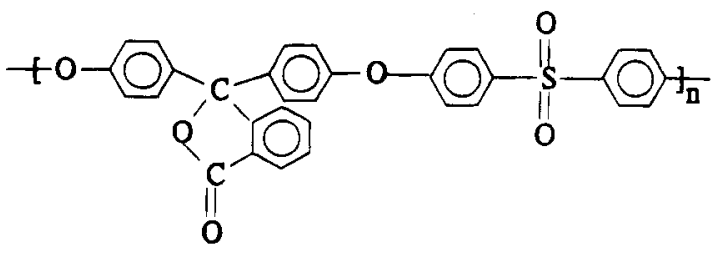

was kindly supplied by the Polymer Physics Laboratory, Changchun Institute of Applied Chemistry, Academia Sinica, China. PES-C was made from a one-step polycondensation of phenolphthalein with 4,4'-dichloro-1,1'-sulfonyldibenzene in the presence of dry $\mathrm{K}_{2} \mathrm{CO}_{3}$. A high molecular weight PES-C fraction was used in this study. The synthesis details of PES-C can be found elsewhere. ${ }^{5}$ Analytical grade chloroform (Merck) was used as a solvent without further purification.

LLS Instrumentation. A commercial LLS spectrometer (ALV/SP-125 equipped with an ALV-5000 multi- $\tau$ digital time correlator) was used with a solid-state laser (ADLAS DPY425II, output power $\approx 400 \mathrm{~mW}$ at $\lambda=532 \mathrm{~nm}$ ) as the light source. The incident beam was vertically polarized with respect to the scattering plane. In our setup, $\beta$ in eq 3 was $\sim 0.9$, a rather high value for an LLS spectrometer capable of doing both static and dynamic LLS simultaneously. Due to this high $\beta$ value, we were able to carry out dynamic LLS in very dilute solutions with a good signal-to-noise ratio, so that the concentration correction was reduced to the minimum. After some proper modifications, ${ }^{10}$ the present LLS spectrometer was capable of doing both static and dynamic LLS continuously in the scattering angle range $5.7-154^{\circ}$. Later on, we will show that the accessible small angle range used in this study was vital for the successful characterization of the large PES-C clusters.

Refractive Index Increment. A precise value of the differential refractive index increment $(\mathrm{d} n / \mathrm{d} C)$ is very essential for the static LLS characterization of polymers. All $\mathrm{d} n / \mathrm{d} C$ values were determined by using a novel differential refractometer, ${ }^{11}$ which was incorporated into our LLS spectrometer where the same laser light source was used in both LLS and the refractometer. In this way, the wavelength correction of the measured $\mathrm{d} n / \mathrm{d} C$ will not be necessary. In addition, a precise detection system was realized by using a $6-\mathrm{mm}$ position sensitive detector (Hamamatsu) and a 16-bits analog-to-digital data acquisition card (National Instrument) to monitor the laser light beam shift caused by the refractive index difference between the solution and solvent. In this study, the differential refractometer was used not only for the measurement of $\mathrm{d} n / \mathrm{d} C$, but also for a precise determination of the PES-C concentration in a given solution.

\section{Results and Discussion}

Figure 1 shows a typical Zimm plot of PEB-C in chloroform at $T=25^{\circ} \mathrm{C}$. The concentration of PES-C ranges from 0.373 to $1.86 \mathrm{mg} / \mathrm{mL}$. According to eq 1 , the extrapolations of $C \rightarrow 0$ and $q \rightarrow 0$ lead to $M_{\mathrm{w}}$, listed as $M_{\mathrm{w}}^{\mathrm{m}}$ in Table 1 . In addition, the slopes of plots of

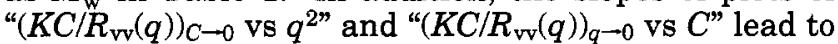
$\left\langle R_{\mathrm{g}}\right\rangle=27.3 \mathrm{~nm}$ and $A_{2}=-2.58 \times 10^{-4}(\mathrm{~mol} \cdot \mathrm{mL}) / \mathrm{g}^{2}$, respectively. The negative value of $A_{2}$ indicates that chloroform is a poor solvent of PES-C. $M_{\mathrm{w}}$ is higher 
Table 1. Mixing Details of Three Polystyrene Mixtures and the LLS Results of These Polystyrene Mixtures and PES-Ca,b

\begin{tabular}{cccccccccc}
\hline sample & $10^{-5} M_{\mathrm{w}, \mathrm{L}}^{\mathrm{m}}$ & $10^{-5} M_{\mathrm{w}, \mathrm{H}}^{\mathrm{m}}$ & $x_{\mathrm{L}}^{\mathrm{m}}$ & $A_{\mathrm{r}}^{\mathrm{c}}$ & $10^{-5} M_{\mathrm{w}}^{\mathrm{c}}$ & $10^{-5} M_{\mathrm{w}}^{\mathrm{m}}$ & $A_{\mathrm{r}}^{\mathrm{m}}$ & $10^{-5} M_{\mathrm{w}, \mathrm{L}}^{\mathrm{c}}$ & $10^{-5} M_{\mathrm{w}, \mathrm{H}}^{\mathrm{c}}$ \\
\hline MX-11 & 3.00 & 59.4 & 0.954 & 1.05 & 5.60 & 5.60 & 1.02 & 2.95 \\
MX-12 & 1.19 & 59.4 & 0.963 & 0.53 & 3.34 & 3.32 & 0.52 & 1.27 & 60.6 \\
MX-21 & 1.19 & 59.4 & 0.991 & 2.10 & 1.75 & 1.73 & 2.05 & 1.19 & 57.4 \\
PES-C & $1.03^{\mathrm{c}}$ & & $0.936^{d}$ & & & 2.00 & 1.01 & 1.07
\end{tabular}

${ }^{a} M_{\mathrm{w}}$ is the weight average molecular weight, $x_{\mathrm{L}}$ is the weight fraction of low molecular weight polymer, and $A_{\mathrm{r}}$ is the ratio of the light intensity scattered from high molecular weight polymer to that from low molecular weight polymer, where the superscripts " $\mathrm{m}$ " and " $\mathrm{c}$ " denote the measured and calculated values and the subscripts " $L$ " and " $H$ " denote low and high molecular weight polymers, respectively. ${ }^{b}$ Relative errors: $M_{\mathrm{w}}, \pm 2 \% ; x_{\mathrm{L}}, \pm 1 \%, A_{\mathrm{r}}, \pm 5 \%{ }^{c}{ }^{c}$ The static LLS result from the PES-C solution after removing the clusters by a $0.1-\mu \mathrm{m}$ filter. ${ }^{d}$ It was determined by using a very precise differential refractometer described in the text.

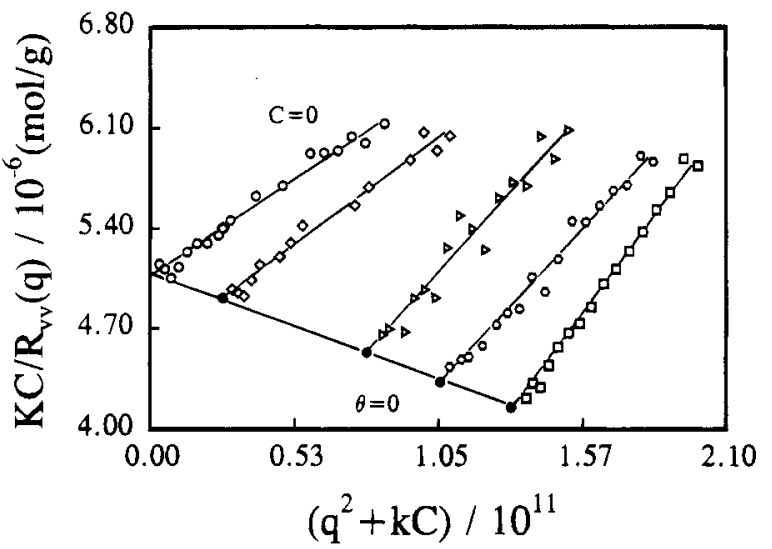

Figure 1. Typical Zimm plot of PES-C in chloroform at $T=$ $25^{\circ} \mathrm{C}$, where $C$ ranges from $3.73 \times 10^{-4}$ to $1.86 \times 10^{-3} \mathrm{~g} / \mathrm{mL}$.

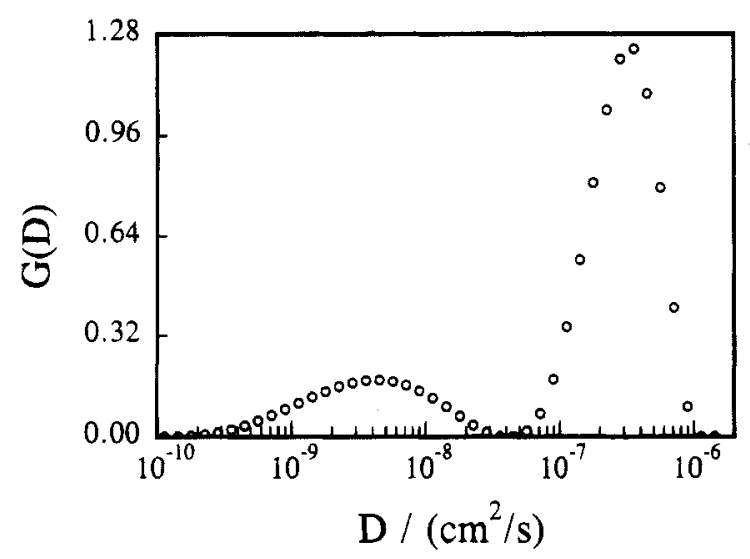

Figure 2. Translational diffusion coefficient distribution $G(D)$ of PES-C in chloroform at $T=25^{\circ} \mathrm{C}$ and $\theta=20^{\circ}$.

than the expected value for a polymer made from polycondensation. In a previous static LLS study of a similar system, ${ }^{5}$ no explanation was given to the unexpected higher value of $M_{\mathrm{w}}$.

Figure 2 shows a typical translational diffusion coefficient distribution $G(D)$ of PES-C in chloroform at $T=$ $25^{\circ} \mathrm{C}$ and $\theta=20^{\circ}$, where $G(D)$ was calculated from time correlation function $G^{(2)}(t, q)$ using the Laplace inversion program CONTIN ${ }^{12}$ equipped with the digital time correlator. The peak with a larger average diffusion coefficient $\langle D\rangle$ is related to linear PES-C chains, and the unexpected second peak with a smaller $\langle D\rangle$ seems to represent the contribution from some larger species with an unknown nature and structure. It should be stated that the existence of these two distinct peaks in $G(D)$ were persistent even in a very dilute solution $(\sim 0.04$ $\mathrm{mg} / \mathrm{mL})$ or at a higher temperature $\left(80^{\circ} \mathrm{C}\right)$.

Figure 3 shows plots of the average hydrodynamic radius $\left\langle R_{\mathrm{h}}\right\rangle$ versus $q^{2}$ for the low molecular weight PES-C chains $(O)$ and high molecular weight clusters ( $\square$ ), where $R_{\mathrm{h}}$ was calculated from $D$ using the Stokes-

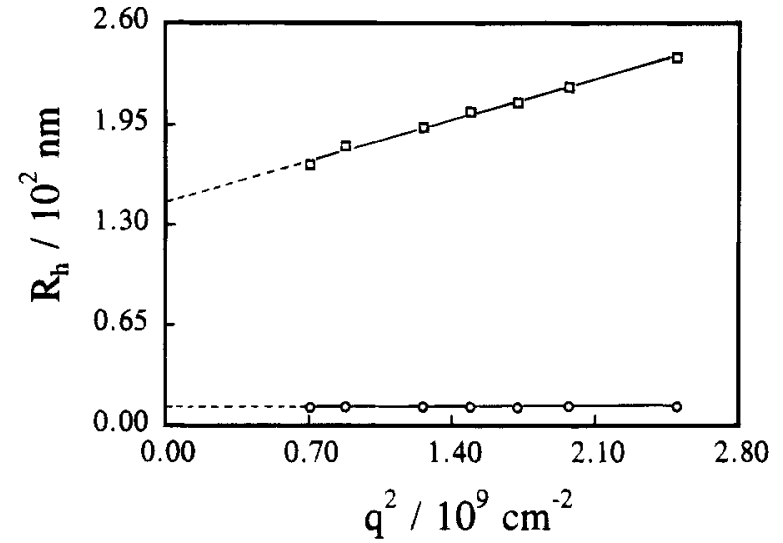

Figure 3. Plots of the average hydrodynamic radius $\left(\left\langle R_{h}\right\rangle\right)$ vs $q^{2}$ for the low molecular weight PES-C chains (O) and high molecular weight clusters $(\square)$.

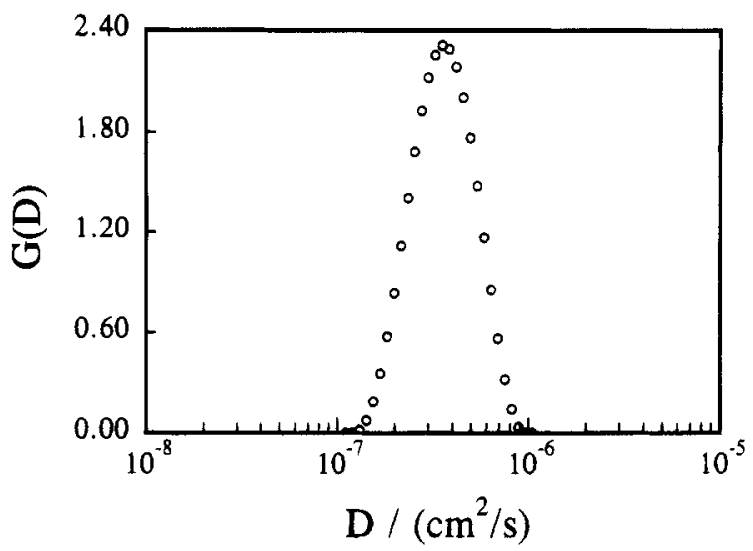

Figure 4. Translational diffusion coefficient distribution $G(D)$ of PES-C after the larger species were removed from the solution by a $0.1-\mu \mathrm{m}$ filter. All experimental conditions are identical to those in Figure 2.

Einstein equation: $R_{\mathrm{h}}=k_{\mathrm{B}} T /(6 \pi \eta D)$ with $k_{\mathrm{B}}, T$, and $\eta$ being the Boltzmann constant, temperature $(K)$ and the solvent viscosity, respectively. $\left\langle R_{\mathrm{h}}\right\rangle$ of the low molecular weight PES-C chains is independent of $q$ because the chain size is very small in comparison with the wavelength. In contrast, $\left\langle R_{\mathrm{h}}\right\rangle$ of the clusters is a linear function of $q^{2}$. As $q \rightarrow 0,\left\langle R_{\mathrm{h}}\right\rangle$ of the clusters approaches $146 \mathrm{~nm}$, which is quite larger than that of the linear PES-C chains. In a previous study of a similar system, ${ }^{5}$ these larger species were overlooked. Further, we found that the larger species can be easily removed from the solution by filtration.

Figure 4 shows a typical translational diffusion coefficient distribution $G(D)$ after the larger clusters were removed from the solution by a filter with a pore size of $0.1 \mu \mathrm{m}$, where experimental conditions identical to those in Figure 2 were used. A comparison of Figures 2 and 4 shows that the larger species have been removed. Moreover, these larger species did not reappear in the 


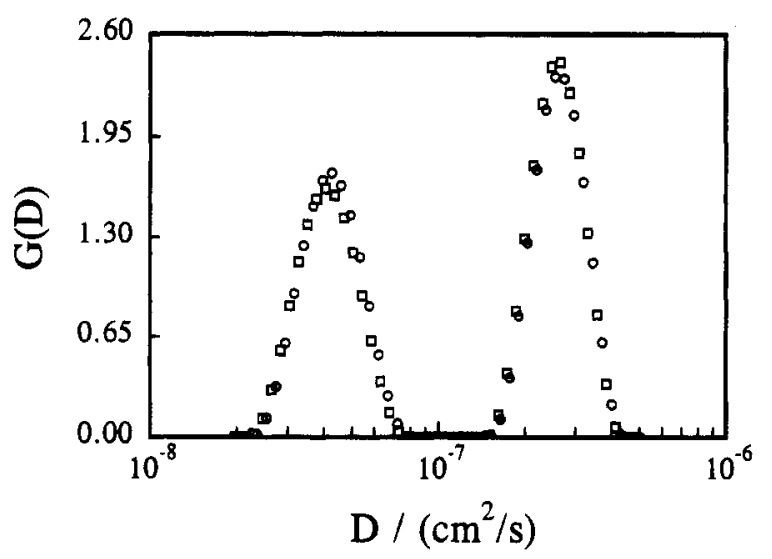

Figure 5. Translational diffusion coefficient distribution $G(D)$ of the polystyrene mixture $\mathrm{MX}-11$ at two scattering angles: (O) $14^{\circ} ;(\square) 17^{\circ}$.

solution even after 6 weeks of standing at room temperature. These larger species could be either the aggregates made of the linear PES-C chains or the highly branched polymer clusters formed during polymerization. However, we believe that they are polymer clusters. A possible way to form the larger clusters could be through the opening of the ester ring in phenolphthalein. This ring opening will lead to branching along the PES-C backbone chain or even possible cross-linking between different PES-C chains. The existence of these larger polymer clusters partially explains why the viscoelastic and mechanical properties of PES-C are similar to those of PEEK even though the expected interactions among the PES-C chains are much less than those among the PEEK chains because the introduction of the phenolphthalein side groups along the PES-C backbone chain prevents a close chain packing. The investigation of the structural detail of the PES-C clusters is ongoing, which is however not the problem we intend to address here.

After removing the larger clusters from the PES-C solution by filtration, we characterized the linear PES-C chains remaining in the solution by static LLS. We found $M_{\mathrm{w}}=1.03 \times 10^{5}$, listed in Table 1 as $M_{\mathrm{w}, \mathrm{L}}^{\mathrm{m}},\left\langle R_{\mathrm{g}}\right\rangle=$ $17.4 \mathrm{~nm}$, and $A_{2}=-4.0 \times 10^{-4}(\mathrm{~mol} \cdot \mathrm{mL}) / \mathrm{g}^{2}$. The value of $\left\langle R_{\mathrm{g}}\right\rangle\left\langle\left\langle R_{\mathrm{h}}\right\rangle \sim 1.34\right.$ shows that the PES-C chain has a random-coil conformation in chloroform at $25^{\circ} \mathrm{C},{ }^{13}$ which has also been observed in a previous intrinsic viscosity study of a similar system. ${ }^{5}$ This coil conformation is expected because there are two very flexible ether linkages (-O-) in each repeating unit of PES-C. The fact that PES-C is a mixture of individual linear chains and clusters triggered us to use the described LLS procedure to reexamine the previous results obtained from Figure 2. Before using it, we decided to check its validity and limitation in the characterization of such a polymer mixture. We used three two-polystyrene mixtures as model systems, wherein the polystyrene with a higher molecular weight acted as the cluster.

Figure 5 shows typical diffusion coefficient distributions of the polystyrene mixture MX-11 at two different scattering angles $\left(O, \theta=14^{\circ}\right.$ and $\left.\square, \theta=17^{\circ}\right)$. The two relaxation processes related to the low and high molecular weight polystyrenes are well separated. The peak with a higher $\langle D\rangle$ shows the polystyrene with a lower molecular weight $\left(M_{\mathrm{w}, \mathrm{L}}\right)$, and the peak with a lower $\langle D\rangle$, the polystyrene with a higher molecular weight $\left(M_{\mathrm{w}, H}\right)$. The narrow widths of the two peaks confirm that the polystyrenes used in this study were narrowly distributed. The positions of the two peaks are basically

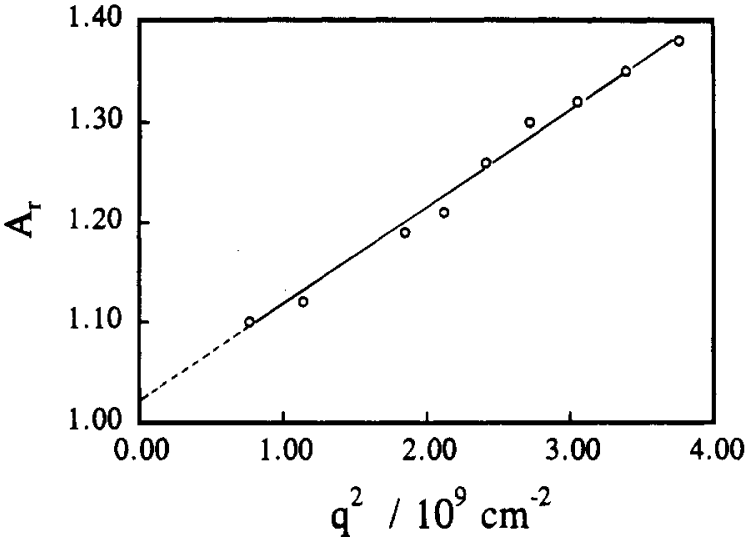

Figure 6. Area ratio $\left(A_{r}=A_{L} / A_{H}\right)$ of the two peaks in Figure 5 as a function of $q^{2}$, where $A_{\mathrm{L}}$ and $A_{\mathrm{H}}$ are the areas under $G(D)$ of the low and high molecular weight polystyrenes in the mixture, respectively.

independent of the scattering angle in the range $9-20^{\circ}$, but the area ratio $A_{\mathrm{r}}\left(=A_{\mathrm{I}} / A_{\mathrm{H}}\right)$ slightly varies.

Figure 6 shows the ratio of the area under the two peaks in Figure 5 as a function of $q^{2}$, where $A_{\mathrm{r}}=A_{\mathrm{L}}$ / $A_{\mathrm{H}}$. As expected, $A_{\mathrm{r}}$ increases with $q^{2}$ because the scattering intensity from the large "cluster" (polystyrene with a higher molecular weight) decreases much faster as $q$ increases than that from the polystyrene with a lower molecular weight. More specifically, on the basis of eqs 1 and 6 , the $q^{2}$ dependence of the scattered light intensity or the peak area $(A)$ under $G(D)$ for a dilute solution at finite $q$, with $\left\langle R_{\mathrm{g}}{ }^{2}\right\rangle q^{2} \ll 1$, can be written as

$$
\begin{aligned}
A=\int_{0}^{\infty} G(D) \mathrm{d} D \propto & R_{\mathrm{vv}}(q) \propto \\
& M_{\mathrm{w}} C /\left(1+\frac{1}{3}\left\langle R_{\mathrm{g}}{ }^{2}\right\rangle q^{2}+2 A_{2} M_{\mathrm{w}} C\right)
\end{aligned}
$$

so that for a polymer mixture of low molecular weight linear chains and high molecular weight clusters the area ratio $\left(A_{\mathrm{r}}\right)$ should be

$$
\begin{aligned}
A_{\mathrm{r}}= & \frac{A_{\mathrm{L}}}{A_{\mathrm{H}}}= \\
& \quad \frac{M_{\mathrm{w}, \mathrm{L}} C_{\mathrm{L}}}{M_{\mathrm{w}, \mathrm{H}} C_{\mathrm{H}}} \frac{\left[1+(1 / 3)\left\langle R_{\mathrm{g}}{ }^{2}\right\rangle_{\mathrm{H}} q^{2}+2 A_{2, \mathrm{H}} M_{\mathrm{w}, \mathrm{H}} C_{\mathrm{H}}\right]}{\left[1+(1 / 3)\left\langle R_{\mathrm{g}}{ }^{2}\right\rangle_{\mathrm{L}} q^{2}+2 A_{2, \mathrm{~L}} M_{\mathrm{w}, \mathrm{L}} C_{\mathrm{L}}\right]} \\
& \cong \frac{M_{\mathrm{w}, \mathrm{L}} C_{\mathrm{L}}}{M_{\mathrm{w}, \mathrm{H}} C_{\mathrm{H}}}\left[a_{\mathrm{L}} a_{\mathrm{H}}+\frac{1}{3}\left(a_{\mathrm{L}}\left\langle R_{\mathrm{g}}{ }^{2}\right\rangle_{\mathrm{H}}-a_{\mathrm{H}}\left\langle R_{\mathrm{g}}{ }^{2}\right\rangle_{\mathrm{L}}\right) q^{2}+o\left(q^{4}\right)\right]
\end{aligned}
$$

where $a_{\mathrm{L}}=1-2 A_{2, \mathrm{~L}} M_{\mathrm{w}, \mathrm{L}} C_{\mathrm{L}}$ and $a_{\mathrm{H}}=1+2 A_{2, \mathrm{H}} M_{\mathrm{w}, \mathrm{H}} C_{\mathrm{H}}$. The plot of " $A_{\mathrm{r}}$ vs $q^{2 \text { " is a straight line when }\left\langle R_{\mathrm{g}}{ }^{2}\right\rangle_{\mathrm{H}} q^{2} \ll}$ 1 . This is why the accessible small angle range used in this study is vital, since $\left\langle R_{\mathrm{g}}{ }^{2}\right\rangle_{\mathrm{H}}$ is usually quite large for clusters with a high average molecular weight. On the basis of eq 9, the intercept in Figure 6 should be very close to $\left(M_{\mathrm{w}, \mathrm{L}} x_{\mathrm{L}}\right) /\left(M_{\mathrm{w}, \mathrm{H}} x_{\mathrm{H}}\right)$ since $a_{\mathrm{L}} a_{\mathrm{H}} \cong 1$ for a very dilute solution; and the slope can be directly related to $\left\langle R_{\mathrm{g}}{ }^{2}\right\rangle_{\mathrm{H}}$ since $\left\langle R_{\mathrm{g}}{ }^{2}\right\rangle_{\mathrm{H}} \gg\left\langle R_{\mathrm{g}}{ }^{2}\right\rangle_{\mathrm{L}}$.

A combination of $\left(A_{\mathrm{r}}\right)_{q \rightarrow 0}\left[=\left(M_{\mathrm{w}, \mathrm{L}} x_{\mathrm{L}}\right) /\left(M_{\mathrm{w}, \mathrm{H}} x_{\mathrm{H}}\right)\right]$ from dynamic LLS with $M_{\mathrm{w}, \text { app }}$ from static LLS leads to $M_{\mathrm{w}, \mathrm{L}} x_{\mathrm{L}}$ and $M_{\mathrm{w}, \mathrm{H}} x_{\mathrm{H}}$. As previously stated, by knowing any one of the four parameters $\left(M_{\mathrm{w}, \mathrm{L}}, x_{\mathrm{L}}, M_{\mathrm{w}, \mathrm{H}}\right.$, and $\left.x_{\mathrm{H}}\right)$, we will be able to calculate the other three. However, it would be difficult to accurately determine $x_{\mathrm{H}}$ or $M_{\mathrm{w}, \mathrm{H}}$ because the amount of the high molecular weight 


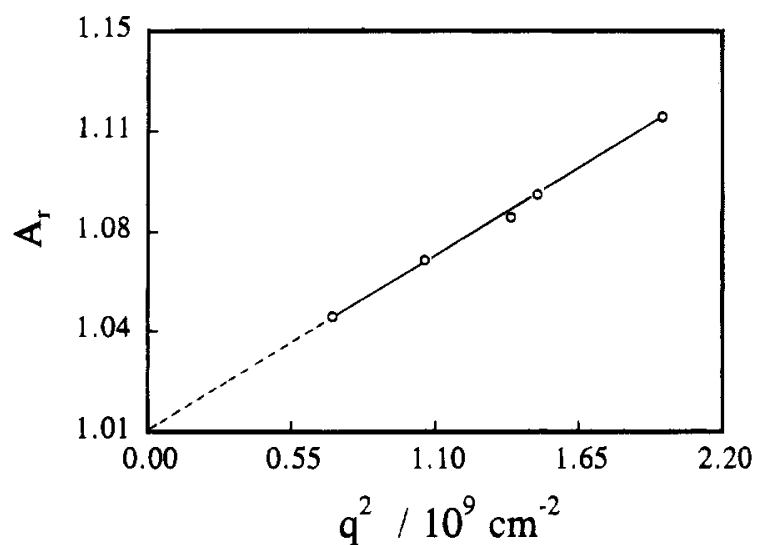

Figure 7. Plot of $A_{r}$ vs $q^{2}$ for PES-C in chloroform at $25^{\circ} \mathrm{C}$, where $O$ represents the experimental data and the line represents the fitting of $A_{\mathrm{r}}=1.01+5.60 \times 10^{-11} q^{2}$.

polymer clusters is usually very small. Therefore, a more practical way is to determine either $M_{\mathrm{w}, \mathrm{L}}$ or $x_{\mathrm{L}}$. Actually, to measure $x_{\mathrm{L}}$, rather than $M_{\mathrm{w}, \mathrm{L}}$, is experimentally preferred due to the higher accuracy and less difficulty. On the basis of this consideration, we used the values of $x_{\mathrm{L}}$ from the preparation of the polymer mixtures to calculate both $M_{\mathrm{w}, \mathrm{L}}^{\mathrm{c}}$ and $M_{\mathrm{w}, \mathrm{H}}^{\mathrm{c}}$.

Table 1 summarizes all calculated values of $M_{\mathrm{w}, \mathrm{L}}^{\mathrm{c}}$ and $M_{\mathrm{w}, \mathrm{H}}^{\mathrm{c}}$ for the three polystyrene mixtures. A comparison of each pair of the corresponding values of " $M_{\mathrm{w}, \mathrm{L}}^{\mathrm{m}}$ and $M_{\mathrm{w}, \mathrm{L}}^{\mathrm{c}}$ " and " $M_{\mathrm{w}, \mathrm{H}}^{\mathrm{m}}$ and $M_{\mathrm{w}, \mathrm{H}}^{\mathrm{c}}$ " for each of the three mixtures shows that the LLS method adopted here works reasonably well for the polystyrene mixtures wherein the high molecular weight polystyrene was used to imitate the large "clusters". The agreement between the measured and calculated weight-average molecular weights gives us confidence to use this method to characterize PES-C. However, it should be stated that if the amount of high molecular weight clusters is too small, i.e., $x_{\mathrm{H}} \ll 1$ or $x_{\mathrm{L}} \sim 1$, the errors introduced in the determination of $x_{\mathrm{H}}$ or the calculation of $M_{\mathrm{w}, \mathrm{H}}$ from the $x_{\mathrm{L}}$ value would be rather large. Fortunately, we can see in Figure 2 that the amount of the clusters in PES-C (i.e., the area under the peak with a lower value of $D$ ) is not too small.

Figure 7 shows a plot of $A_{\mathrm{r}}$ versus $q^{2}$ for PES-C in chloroform at $T=25^{\circ} \mathrm{C}$, where " $O$ " is the experimental data and the line is a least-squares fitting of $A_{\mathrm{r}}=1.01$ $+5.60 \times 10^{-11} q^{2}$. On the basis of eq 9 , we got $\left(M_{\mathrm{w}, L} x_{\mathrm{L}}\right) /$ $\left(M_{\mathrm{w}, \mathrm{H} x_{\mathrm{H}}}\right) \cong 1.01$ from the intercept and $\left\langle R_{\mathrm{g}}\right\rangle$ (the radius of gyration of the clusters) $\cong 130 \mathrm{~nm}$ from the slope, respectively, where $a_{\mathrm{L}}$ and $a_{\mathrm{H}}$ were corrected with the known $A_{2}$ values. It should be noted that $\left\langle R_{\mathrm{g}}\right\rangle /\left\langle R_{\mathrm{h}}\right\rangle$ for the PES-C clusters is only $\sim 0.89$, which implies that the PES-C clusters might be the highly branched polymer clusters ${ }^{14}$ and have a more compact conformation in chloroform at $T=25^{\circ} \mathrm{C} .{ }^{13}$ From $M_{\mathrm{w}}^{\mathrm{m}}$ and $A_{\mathrm{r}}^{\mathrm{m}}$ listed in Table 1 , we calculated $M_{\mathrm{w}, \mathrm{L}} x_{\mathrm{L}}$ and $M_{\mathrm{w}, \mathrm{H}} x_{\mathrm{H}}$. Further, we used the new differential refractometer ${ }^{11}$ to determine $x_{\mathrm{L}}$

Figure 8 shows a plot of the refractive index increment $(\Delta n)$ versus $C$ for PES-C in chloroform at $T=25$ ${ }^{\circ} \mathrm{C}$, where $\mathrm{O}$ is the experimental data and the line shows a least-squares fitting of $\Delta n=-8.34 \times 10^{-7}+2.04 \times$ $10^{-1} C \cong 2.04 \times 10^{-1} C$. The slope leads to the value of $\mathrm{d} n / \mathrm{d} C=0.204 \mathrm{~mL} / \mathrm{g}$, which was used in Figure 1 to calculate $M_{\mathrm{w}}^{\mathrm{m}}$. With this calibration between $\Delta n$ and $C$, we are able to determine the concentration of a given PES-C solution as long as the same solvent and tem-

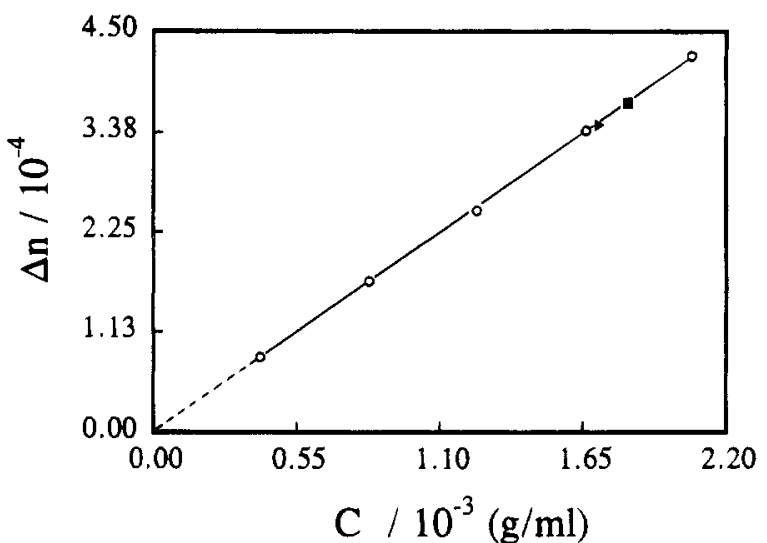

Figure 8. Plot of the refractive index increment $(\Delta n)$ vs $C$ for PES-C in chloroform at $T=25^{\circ} \mathrm{C}$, where $O$ represents the experimental data and the line, a least-squares fitting of $\Delta n$ $\cong 0.204 C$. and $\triangle$ are the results obtained before and after the large clusters are removed from the PES-C solution with a $0.1-\mu \mathrm{m}$ filter.

perature are used. Figure 4 has shown that the large clusters in PES-C can be easily removed by a filter with a pore size of $0.1 \mu \mathrm{m}$. Thus, for a given solution, we can determine $C$ and $C_{\mathrm{L}}$, respectively, from $\Delta n$ measured before and after the filtration. Figure 8 shows how $C(\square)$ and $C_{\mathrm{L}}$ can be found from the values of $\Delta n$ determined before and after the filtration. The values of $C_{\mathrm{I}} / C$ are listed in Table 1 under $x_{\mathrm{L}}^{\mathrm{m}}$. Figure 8 also shows that in the dilute range the plot of $\Delta n$ vs $C$ is a straight line; namely $\Delta n$ is proportional to $C$. Therefore, $x_{\mathrm{L}}$ can be directly calculated from the ratio of the $\Delta n$ values. In this way, $x_{\mathrm{L}}$ is independent of the calibration of " $\Delta n$ vs $C$ " and the uncertainties in $x_{\mathrm{L}}$ were greatly reduced, as also for the final results.

With the value of $x_{L}$, we found the weight-average molecular weights of the PES-C linear chains and clusters, respectively, listed as $M_{\mathrm{w}, \mathrm{L}}^{\mathrm{c}}$ and $M_{\mathrm{w}, \mathrm{H}}^{\mathrm{c}}$ in Table 1. For PES-C, $M_{\mathrm{w}, \mathrm{L}}^{\mathrm{c}}$ is close to $M_{\mathrm{w}, \mathrm{L}}^{\mathrm{m}}$, which shows the LLS method used in this study is a good, workable, and useful method for the characterization of a polymer mixture made of linear chains and clusters.

\section{Conclusions}

The method of combining the weight-average molecular weight $\left(M_{\mathrm{w}}\right)$ from static laser light scattering (LLS) and the area ratio $\left(A_{\mathrm{r}}\right)$ under the translational diffusion coefficient distribution $(G(D))$ peaks from dynamic LLS is useful for the characterization of a polymer mixture made of individual chains and clusters. Using this method, we have accomplished the characterization of a recently developed high-performance thermoplastic resin: phenolphthalein poly(ether sulfone) (PES-C). We found that PES-C consists of $\sim 94 \%$ (by weight) linear polymer chains and $\sim 6 \%$ large polymer clusters. The weight-average molecular weight $\left(M_{\mathrm{w}}\right)$ of the linear PES-C chains is $\sim 100000$ and the $M_{\mathrm{w}}$ of the clusters is $\sim 15$ times higher. The existence of these high molecular weight clusters might partially explain the high glass transition temperature and other viscoelastic properties of PES-C. The linear PES-C chains have a typical coil conformation in chloroform at room temperature, while the PES-C clusters seem to have a more compact structure in the solution. It is expected that the LLS method used here will be particularly useful in the characterization of association/aggregation in a given polymer or colloidal system. 
Acknowledgment. The financial support of this work by the RGC (the Research Grants Council of the Hong Kong Government) Earmarked Grant 1994/95 (CUHK 299/94P, 221600260) is gratefully acknowledged.

\section{References and Notes}

(1) Wu, C.; Zuo, J.; Chu, B. Macromolecules 1989, 22 (2), 633.

(2) Wu, C. J. Appl. Polym. Sci. 1994, 54, 969.

(3) Zimm, B. H. J. Chem. Phys. 1948, 16, 1099.

(4) Debye, P. J. Phys. Colloid Chem. 1947, 51, 18.

(5) Bo, S.; Yang, H.; Chen, T. Functional Polym. 1991, 4, 147.

(6) Zhou, Z. K.; Peiffer, D. G.; Chu, B. Macromolecules 1994, 27, 1428.
(7) Pecora, R. Dynamic Light Scattering; Plenum Press: New York, 1976.

(8) Chu, B. Laser Light Scattering; Academic Press: New York, 1974.

(9) Stockmayer, W. H.; Schmidt, M. Pure Appl. Chem. 1982, 54, 407; Macromolecules 1984, 17, 509.

(10) Wu, C.; Chan, K. K.; Xia, K. Q. Macromolecules 1995, 28, 1032.

(11) Wu, C.; Xia, K. Q. Rev. Sci. Instrum. 1993, 65, 587.

(12) Provencher, S. W. Biophys. J. 1976, 16, 29; J. Chem. Phys. 1976, 64, 2772; Makromol. Chem. 1979, 180, 201.

(13) Stockmayer, W. H.; Schmidt, M. Pure Appl. Chem. 1982, 54, 407; Macromolecules 1984, 17, 509.

(14) Wu, C.; Zuo, J.; Chu, B. Macromolecules 1989, 22, 633.

MA950224F 